\title{
On a translated sum over primitive sequences related to a conjecture of Erdôs
}

\author{
Nadir Rezzoug ${ }^{1}$, Ilias Laib ${ }^{2}$ and Guenda Kenza ${ }^{3}$ \\ ${ }^{1}$ Laboratory of Analysis and Control of Partial Differential Equations \\ Faculty of Exact Sciences, Djillali Liabes University \\ Sidi Bel Abbes, Algeria \\ e-mail: nadir793167115@gmail.com \\ ${ }^{2}$ ENSTP, Garidi Kouba, 16051, Algiers, Algeria \\ and Laboratory of Equations with Partial Non-Linear Derivatives \\ ENS Vieux Kouba, Algiers, Algeria \\ e-mail: laib23@yahoo.fr \\ ${ }^{3}$ Faculty of Mathematics, University of Sciences and Technology Houari Boumédiène, \\ Algiers, Algeria \\ e-mail: ken.guenda@gmail.com
}

Received: 22 November 2019

Revised: 6 October 2020

Accepted: 14 October 2020

Abstract: For $x$ large enough, there exists a primitive sequence $\mathcal{A}$, such that

$$
\sum_{a \in \mathcal{A}} \frac{1}{a(\log a+x)} \gg \sum_{p \in \mathcal{P}} \frac{1}{p(\log p+x)},
$$

where $\mathcal{P}$ denotes the set of prime numbers.

Keywords: Primitive sequences, Erdős conjecture, Prime numbers.

2010 Mathematics Subject Classification: 11Bxx.

\section{Introduction}

A sequence $\mathcal{A}$ of positive integers is said to be primitive if there is no element of $\mathcal{A}$ which divides any other. We can see directly that the set of primes $\mathcal{P}=\left(p_{n}\right)_{n \geq 1}$ is primitive, as well as the sequences of the form:

$$
\mathcal{A}_{d}^{k}=\left\{p_{1}^{\alpha_{1}} p_{2}^{\alpha_{2}} \ldots p_{k}^{\alpha_{k}} ; \alpha_{1}, \ldots, \alpha_{k} \in \mathbb{N}, \alpha_{1}+\ldots+\alpha_{k}=d\right\} .
$$


Erdős [2] showed that for a primitive sequence $\mathcal{A} \neq\{1\}$, the series $\sum_{a \in \mathcal{A}} \frac{1}{a \log a}$ converges. Later, in [3], he conjectured that if $\mathcal{A} \neq\{1\}$ is a primitive sequence, then $\sum_{a \in \mathcal{A}} \frac{1}{a \log a} \leq \sum_{p \in \mathcal{P}} \frac{1}{p \log p}$. Recently, in $[4,5]$, the authors studied a translated sums of the form

$$
S(\mathcal{A}, x)=\sum_{a \in \mathcal{A}} \frac{1}{a(\log a+x)},
$$

where $x \in \mathbb{R}_{+}$. In [5], the authors constructed a primitive sequence $\mathcal{A}$, such that for all $x \geq 81$ $S(\mathcal{A}, x)>S(\mathcal{P}, x)$. In this note, we prove the following result.

Theorem 1.1. Let $\lambda \geq 1$ and $t>0$, then for any $x \geq 1656 \lambda^{2 t}\left(\log \left(\lambda^{2 t}+2\right)\right)^{3 / 2}$, there exists a primitive sequence $\mathcal{A}$ such that

$$
S(\mathcal{A}, x) \geq \lambda^{t} S(\mathcal{P}, x) .
$$

For a real $x$, the quantity $\lfloor x\rfloor$ denotes the integer part of $x$.

\section{Lemmas}

Lemma $2.1([6,8])$. We have:

$$
\begin{array}{rlrl}
p_{n} & \geq n \log n & (\forall n \geq 2) \\
p_{n} & \leq n(\log n+\log \log n) \quad(\forall n \geq 6) \\
\sum_{p \in \mathcal{P}, p \leq x} \frac{1}{p} & >\log \log x \quad(x>1) .
\end{array}
$$

Lemma 2.2 ([1]). For $k \geq 463$,

$$
p_{k+1} \leq p_{k}\left(1+\frac{1}{2 \log ^{2} p_{k}}\right) .
$$

Lemma 2.3. For any real number $x>0$ and any integer $k \geq 2$ the following holds

$$
\sum_{n>k} \frac{1}{p_{n}\left(\log p_{n}+x\right)} \leq \frac{\log \left(1+\frac{x}{\log k}\right)}{x} .
$$

Proof. Let $x>0$ be a real number and $k \geq 2$ be an integer. By (1) and since the function $t \mapsto \frac{d t}{t \log t(\log t+x)}$ decreases on $[1,+\infty)$, then we obtain:

$$
\begin{aligned}
\sum_{n>k} \frac{1}{p_{n}\left(\log p_{n}+x\right)} & \leq \sum_{n>k} \frac{1}{n \log n(\log n+\log \log n+x)} \\
& \leq \sum_{n>k} \frac{1}{n \log n(\log n+x)} \leq \int_{k}^{+\infty} \frac{d t}{t \log t(\log t+x)}
\end{aligned}
$$

We put $u=\log t$, so

$$
\int_{k}^{+\infty} \frac{d t}{t \log t(\log t+x)}=\int_{\log k}^{+\infty} \frac{d u}{u(u+x)}=\frac{1}{x} \int_{\log k}^{+\infty}\left(\frac{1}{u}-\frac{1}{u+x}\right) d u=\frac{\log \left(1+\frac{x}{\log k}\right)}{x} .
$$

This ends the proof. 
Lemma 2.4. For any integer $n \neq 0$, we have:

$$
n ! \leq n^{n} e^{1-n} \sqrt{n}
$$

Proof. For $n=1$, the inequality is verified. For $n \geq 2$, the result follows from

$$
n ! \leq n^{n} e^{-n} \sqrt{2 \pi n} e^{1 / 12 n}
$$

(see [7]).

\section{Proof of Theorem 1.1}

Let $\lambda \geq 1$ and let $t>0$. To prove this theorem, we need the parameters $\alpha, c$ and $\beta$ which satisfy:

$$
\begin{gathered}
c \alpha \geq e^{\beta}+\log 1.008,0<\alpha \leq \frac{5}{12} \\
\beta \geq 1.950
\end{gathered}
$$

those parameters will be chosen later, the real $c$ is chosen to be the smallest possible value so that; for any $x \geq c \lambda^{2 t}\left(\log \left(\lambda^{2 t}+2\right)\right)^{3 / 2}$, there exists a primitive sequence $\mathcal{A} \neq\{1\}$ such that $\sum_{a \in \mathcal{A}} \frac{1}{a(\log a+x)}>\lambda^{t} \sum_{p \in \mathcal{P}} \frac{1}{p(\log p+x)}$. Let $p_{k}$ be the largest prime satisfying $p_{k} \leq e^{\alpha x}$, then according to Lemma 2.2 and (1), we obtain

$$
p_{k} \leq e^{\alpha x}<p_{k+1}<1.008 p_{k}
$$

Assume that $d=\left\lfloor\beta+\log \lambda^{2 t}+\frac{3}{2} \log \log \left(\lambda^{2 t}+2\right)\right\rfloor$, then from (C1) and (C2), we have $x \geq \frac{1}{\alpha}\left(e^{d}+\log 1.008\right)$ and from (3) and (4), we obtain

$$
\sum_{n=1}^{k} \frac{1}{p_{n}}>\log \log p_{k}>\log \log \frac{p_{k+1}}{1.008}>\log \log \frac{e^{\alpha x}}{1.008} \geq d .
$$

Now, we define the following sets of positive integers:

$$
\mathcal{P}^{k}=\left\{p_{n} \mid p_{n} \in \mathcal{P}, p_{n}>p_{k}\right\}, \mathcal{A}=\mathcal{A}_{d}^{k} \cup \mathcal{P}^{k}
$$

It is clear that $\mathcal{A}_{d}^{k} \cap \mathcal{P}^{k}=\varnothing$ and the sets $\mathcal{A}_{d}^{k}, \mathcal{P}^{k}, \mathcal{A}$ are primitive sequences. Then, according to the multinomial formula and (5), we have

$$
\begin{aligned}
\sum_{a \in \mathcal{A}_{d}^{k}} \frac{1}{a} & =\sum_{\alpha_{1}+\ldots+\alpha_{k}=d} \frac{1}{p_{1}^{\alpha_{1}} p_{2}^{\alpha_{2}} \ldots p_{k}^{\alpha_{k}}} \geq \sum_{\alpha_{1}+\ldots+\alpha_{k}=d} \frac{\left(1 / p_{1}\right)^{\alpha_{1}}}{\left(\alpha_{1}\right) !} \ldots \frac{\left(1 / p_{k}\right)^{\alpha_{k}}}{\left(\alpha_{k}\right) !} \\
& =\frac{1}{d !}\left(\sum_{n=1}^{k} \frac{1}{p_{n}}\right)^{d}>\frac{d^{d-1}}{d !} \sum_{n=1}^{k} \frac{1}{p_{n}} .
\end{aligned}
$$

So,

$$
\sum_{a \in \mathcal{A}_{d}^{k}} \frac{1}{a}>\frac{d^{d-1}}{d !} \sum_{n=1}^{k} \frac{1}{p_{n}}
$$


Since $x \geq c \lambda^{2 t}\left(\log \left(\lambda^{2 t}+2\right)\right)^{3 / 2}$, then from (C1) and (C2) we obtain $e^{\alpha x} \geq 3303 \geq p_{464}$. Hence using (4), we find $p_{464} \leq p_{k} \leq e^{\alpha x}<p_{k+1}<1.008 p_{k}$. By using (2), we get

$$
\log p_{k} \leq \alpha x \leq \log p_{k}+\log 1.008 \leq \log (k(\log k+\log \log k))+\log 1.008
$$

Now, since the function

$$
t \mapsto \frac{\log (t(\log t+\log \log t))+\log 1.008}{\log t}
$$

decreases on $[464,+\infty)$, then we have

$$
\frac{\log (t(\log t+\log \log t))+\log 1.008}{\log t} \leq \frac{\log (464(\log 464+\log \log 464))+\log 1.008}{\log 464} \simeq 1.339
$$

that is,

$$
\alpha x \leq 1.339 \log k .
$$

By using inequality (7) and Lemma 2.3, we find

$$
\sum_{n>k} \frac{1}{p_{n}\left(\log p_{n}+x\right)} \leq \frac{\log \left(1+\frac{x}{\log k}\right)}{x}<\frac{\log \left(1+\frac{1.339}{\alpha}\right)}{x} .
$$

On the other hand, according to (4) and (5), we have for $x \neq 0$

$$
\sum_{n=1}^{k} \frac{1}{p_{n}\left(\log p_{n}+x\right)} \geq \sum_{n=1}^{k} \frac{1}{p_{n}(\alpha x+x)} \geq \frac{1}{(\alpha+1) x} \sum_{n=1}^{k} \frac{1}{p_{n}} \geq \frac{d}{(\alpha+1) x},
$$

and from (8) we obtain

$$
\sum_{n=1}^{k} \frac{1}{p_{n}\left(\log p_{n}+x\right)} \geq \frac{d}{(\alpha+1) \log \left(1+\frac{1.339}{\alpha}\right)} \sum_{n>k} \frac{1}{p_{n}\left(\log p_{n}+x\right)} .
$$

Now we put $h(\alpha)=(\alpha+1) \log \left(1+\frac{1.339}{\alpha}\right)$, then we obtain

$$
\sum_{n=1}^{k} \frac{1}{p_{n}\left(\log p_{n}+x\right)} \geq \frac{d}{h(\alpha)}\left(\sum_{n=1}^{+\infty} \frac{1}{p_{n}\left(\log p_{n}+x\right)}-\sum_{n=1}^{k} \frac{1}{p_{n}\left(\log p_{n}+x\right)}\right),
$$

therefore,

$$
\left(1+\frac{d}{h(\alpha)}\right) \sum_{n=1}^{k} \frac{1}{p_{n}\left(\log p_{n}+x\right)} \geq \frac{d}{h(\alpha)} \sum_{n=1}^{+\infty} \frac{1}{p_{n}\left(\log p_{n}+x\right)} .
$$

Thus

$$
\sum_{n=1}^{k} \frac{1}{p_{n}\left(\log p_{n}+x\right)} \geq \frac{d}{d+h(\alpha)} \sum_{n=1}^{+\infty} \frac{1}{p_{n}\left(\log p_{n}+x\right)} .
$$

Since $p_{k}^{d}$ is the largest element in $\mathcal{A}_{d}^{k}$, then according to (4), we have for any $a \in \mathcal{A}_{d}^{k}$

$$
\log a \leq d \log p_{k} \leq d \alpha x
$$

hence, from (6), we obtain: 


$$
\begin{aligned}
\sum_{a \in \mathcal{A}} \frac{1}{a(\log a+x)} & =\sum_{a \in \mathcal{A}_{d}^{k} \cup \mathcal{P}^{k}} \frac{1}{a(\log a+x)}=\sum_{a \in \mathcal{A}_{d}^{k}} \frac{1}{a(\log a+x)}+\sum_{a \in \mathcal{P}^{k}} \frac{1}{a(\log a+x)} \\
& \geq \frac{1}{(d \alpha x+x)} \sum_{a \in \mathcal{A}_{d}^{k}} \frac{1}{a}+\sum_{n>k} \frac{1}{p_{n}\left(\log p_{n}+x\right)} \\
& >\frac{d^{d-1}}{d !(d \alpha+1)} \sum_{n=1}^{k} \frac{1}{p_{n}\left(\log p_{n}+x\right)}+\sum_{n>k} \frac{1}{p_{n}\left(\log p_{n}+x\right)} \\
& =\left(\frac{d^{d-1}}{d !(d \alpha+1)}-1\right) \sum_{n=1}^{k} \frac{1}{p_{n}\left(\log p_{n}+x\right)}+\sum_{n=1}^{+\infty} \frac{1}{p_{n}\left(\log p_{n}+x\right)}
\end{aligned}
$$

According to (C1), and since the sequence $\left(u_{n}\right)_{n \geq 2}$ where $u_{n}=\frac{n^{n-1}-n !}{n n !}$ increases, then we have $\frac{d^{d-1}}{d !(d \alpha+1)}-1 \geq 0$ for $d \geq 4$. By using this last inequality and (9), we obtain

$$
\left(\frac{d^{d-1}}{d !(d \alpha+1)}-1\right) \sum_{n=1}^{k} \frac{1}{p_{n}\left(\log p_{n}+x\right)} \geq\left(\frac{d^{d-1}}{d !(d \alpha+1)}-1\right) \frac{d}{d+h(\alpha)} \sum_{n=1}^{+\infty} \frac{1}{p_{n}\left(\log p_{n}+x\right)} .
$$

Therefore,

$$
\begin{aligned}
\sum_{a \in \mathcal{A}} \frac{1}{a(\log a+x)} & >\left(\left(\frac{d^{d-1}}{d !(d \alpha+1)}-1\right) \frac{d}{d+h(\alpha)}+1\right) \sum_{n=1}^{+\infty} \frac{1}{p_{n}\left(\log p_{n}+x\right)} \\
& =\frac{d^{d}+d !(d \alpha+1) h(\alpha)}{d !(d \alpha+1)(d+h(\alpha))} \sum_{n=1}^{+\infty} \frac{1}{p_{n}\left(\log p_{n}+x\right)},
\end{aligned}
$$

by applying Lemma 2.4 , we get

$$
\sum_{a \in \mathcal{A}} \frac{1}{a(\log a+x)}>\left(\frac{e^{d-1}+\sqrt{d}(d \alpha+1) h(\alpha)}{\sqrt{d}(d \alpha+1)(d+h(\alpha))}\right) \sum_{n=1}^{+\infty} \frac{1}{p_{n}\left(\log p_{n}+x\right)} .
$$

It follows from the expression of $d$, that: $d>\beta-1+\log \lambda^{2 t}+\frac{3}{2} \log \log \left(\lambda^{2 t}+2\right)$, then $e^{d-1}>e^{\beta-2} \lambda^{2 t}\left(\log \left(\lambda^{2 t}+2\right)\right)^{3 / 2}$.

And since $\log \lambda^{2 t}<\log \left(\lambda^{2 t}+2\right), \log \log \left(\lambda^{2 t}+2\right) \leq \log \left(\lambda^{2 t}+2\right)-1$ and $\beta \geq 1.950$, we have $d<(\beta+1) \log \left(\lambda^{2 t}+2\right)$, then $d \alpha+1<((\beta+1) \alpha+1) \log \left(\lambda^{2 t}+2\right)$.

So, the formula (10) becomes

$$
\sum_{a \in \mathcal{A}} \frac{1}{a(\log a+x)}>j_{\alpha, \beta}(\lambda) \sum_{n=1}^{+\infty} \frac{1}{p_{n}\left(\log p_{n}+x\right)} .
$$

where

$$
j_{\alpha, \beta}(\lambda)=\frac{e^{\beta-2} \lambda^{2 t}+\sqrt{\beta+1}((\beta+1) \alpha+1) h(\alpha)}{\sqrt{\beta+1}((\beta+1) \alpha+1)\left((\beta+1) \log \left(\lambda^{2 t}+2\right)+h(\alpha)\right)} .
$$

Now, we must choose $\alpha$ and $\beta$ so that, for any $\lambda \geq 1$ and any $t>0, j_{\alpha, \beta}(\lambda) \geq 1$ and $\frac{e^{\beta}+\log 1.008}{\alpha}$ is the smallest possible. That is, for any $\lambda \geq 1$ and for any $t>0$

$$
\frac{e^{\beta-2}}{\sqrt{\beta+1}(\beta+1)((\beta+1) \alpha+1)} \geq \frac{\log \left(\lambda^{2 t}+2\right)}{\lambda^{2 t}} .
$$


Since, for any $t>0$ the function $\lambda \mapsto \frac{\log \left(\lambda^{2 t}+2\right)}{\lambda^{2 t}}$ decreases on $[1,+\infty)$, then

$$
\frac{e^{\beta-2}}{\sqrt{\beta+1}(\beta+1)((\beta+1) \alpha+1)} \geq \log 3 .
$$

Hence, $\frac{e^{\beta-2}-(\beta+1)^{\frac{3}{2}} \log 3}{(\beta+1)^{\frac{5}{2}} \log 3} \geq \alpha$ and $\frac{e^{\beta}+\log 1.008}{\alpha} \geq \frac{\left(e^{\beta}+\log 1.008\right)(\beta+1)^{\frac{5}{2}} \log 3}{e^{\beta-2}-(\beta+1)^{\frac{3}{2}} \log 3}$.

Finally, we will choose $\beta$ so that the quantity $\frac{\left(e^{\beta}+\log 1.008\right)(\beta+1)^{\frac{5}{2}} \log 3}{e^{\beta-2}-(\beta+1)^{\frac{3}{2}} \log 3}$ is also the smallest possible.

A computer calculation gives $\beta \simeq 6.264, \alpha \simeq 0.317$ and $c \simeq 1655.234$. By replacing $\alpha$ and $\beta$ in (11), we get

$$
\sum_{a \in \mathcal{A}} \frac{1}{a(\log a+x)}>\frac{71.094 \lambda^{2 t}+19.381}{64.659 \ln \left(\lambda^{2 t}+2\right)+19.381} \sum_{n=1}^{+\infty} \frac{1}{p_{n}\left(\log p_{n}+x\right)} .
$$

But, for every $\lambda \geq 1$ and every $t>0$, we have

$$
\frac{71.094 \lambda^{2 t}+19.381}{64.659 \ln \left(\lambda^{2 t}+2\right)+19.381}>\lambda^{t},
$$

which leads to the inequality our main theorem. Thus, for $\lambda \geq 1, t>0$ and for any $x \geq 1656.3 \lambda^{2 t}\left(\log \left(\lambda^{2 t}+2\right)\right)^{3 / 2}$, since $d=\left\lfloor 6.264+\log \lambda^{2 t}+\frac{3}{2} \log \log \left(\lambda^{2 t}+2\right)\right\rfloor$ and $k$ is the greatest integer such that $p_{k} \leq e^{0.317 x}$, the sequence $\mathcal{A}$ is well defined. This ends the proof.

\section{References}

[1] Dusart., P. (1998). Autour de la fonction qui compte le nombre de nombres premiers, thèse de doctorat, université de Limoges, 17-1998.

[2] Erdős, P. (1935). Note on sequences of integers no one of which is divisible by any other, J.Lond. Math. Soc, 10, 126-128.

[3] Erdős, P., \& Zhang, Z. (1993). Upper bound of $\sum 1 /\left(a_{i} \log a_{i}\right)$ for primitive sequences, Math. Soc, 117, 891-895.

[4] Farhi, B. (2017). Results and conjectures related to a conjecture of Erdôs concerning primitive sequences, arXiv: 1709.08708v2 [math.NT] 25 Sep 2017.

[5] Laib, I., Derbal, A. \& Mechik, R. (2019). Somme translatée sur des suites primitives et la conjecture d'Erdős. C. R. Acad. Sci. Paris, Ser. I, 357, 413-417.

[6] Massias, J.-P., \& Robin, G. (1996). Bornes effectives pour certaines fonctions concernant les nombres premiers, J. Theori. Nombres Bordeaux, 8, 215-242.

[7] Robbins, H. (1955). A remark on Stirling's formula, Amer. Math. Monthly, 62, 26-29.

[8] Rosser, J. B., \& Schoenfeld, L. (1962). Approximates formulas for some functions of prime numbers, Illinois Journal Math, 6, 64-94. 